\title{
Longitudinal Myelitis of a Neuro-Behçet Patient
}

\author{
Qi TANG, Jing TIAN
}

Department of Rheumatology, Second Xiangya Hospital of Central South University, Changsha, Hunan, China

\begin{abstract}
Behçet's disease is a chronic, relapsing inflammatory disorder of unknown etiology. Neuro-Behçet's disease (NBD) occurs in approximately 5 to $49 \%$ of patients with Behçet's disease. Spinal cord involvement is very rare in NBD. In this article, we report a 22-year-old male patient of NBD with longitudinal myelitis involving the entire spinal cord. Patient was admitted with one-week of headache, vomiting, and urinary incontinence. Before admission, he felt motor weakness for two years and had noticed recurrent genital and oral ulcers with a frequency of more than three episodes per year for five years. $T_{2}$-weighted spinal magnetic resonance images showed hyperintensities within the entire spinal cord. He was diagnosed as NBD with longitudinal myelitis. Intravenous methylprednisolone $(120 \mathrm{mg} /$ day $)$ was administered for three days, followed by an oral administration of prednisolone ( $45 \mathrm{mg} /$ day). In conjunction with the steroid therapy, intravenous cyclophosphamide $(0.4 \mathrm{~g} / \mathrm{week})$ was administered twice. Rapid improvements were detected after receiving treatments for half a month. NBD associated longitudinal myelitis is really rare. This case provided important implications for the diagnosis and treatment of longitudinal myelitis in NBD patients.

Keywords: Corticosteroid; cyclophosphamide; longitudinal myelitis; magnetic resonance imaging; neuro-Behçet's disease.
\end{abstract}

Behçet's disease is a chronic, relapsing inflammatory disorder of unknown etiology, characterized by a triad of recurrent aphthous stomatitis, genital ulceration, and uveitis, along with cardiovascular, pulmonary, gastrointestinal, and nervous system involvement. ${ }^{1}$ Neuro-Behçet's disease (NBD) occurs in approximately 5 to $49 \%$ of patients with Behçet's disease. ${ }^{2}$ Although lesions of the brainstem and basal ganglia are most commonly associated with NBD, spinal cord involvement is very rare. Here, we report a case of longitudinal myelitis associated with NBD in a young male patient.

\section{CASE REPORT}

A 22-year-old male patient was admitted with one-week of headache, vomiting, and urinary incontinence. Before admission, he had felt motor weakness for two years and noticed recurrent genital and oral ulcers with a frequency of more than three episodes per year for five years. He had no visual impairment. There were no cutaneous lesions such as papules, macules, ecchymosis or nodules. The pathergy test was positive. On neurological examination, he had impaired muscle strength in the upper (phase-4 on both sides) and lower (phase- 4 in the left and phase- 3 in the right) extremities. His impairment level according to American Spinal Injury Association classification was $\mathrm{D}$. He was able to walk only by assistance from his parents or a walking stick. Pathological reflexes such as the Hoffmann and Babinski signs were present bilaterally. Abdominal reflex, cremasteric reflex, tendon reflexes disappeared bilaterally. He had a sensory level at $\mathrm{C} 2$ on the right and $\mathrm{T}_{2}$ on the left. Routine biochemical tests showed an increased erythrocyte sedimentation rate $(85 \mathrm{~mm} / \mathrm{h})$ and C-reactive protein level (124 mg/L). Serum autoantibody tests were negative for anti-nuclear antibody, anti-aquaporin-4 antibody, and other autoantibodies. His cerebrospinal fluid (CSF)

Received: September 16, 2014 Accepted: October 06, 2015 Published online: November 03, 2015

Correspondence: Jing Tian, MD. Department of Rheumatology, Second Xiangya Hospital of Central South University, 410001 Changsha, Hunan, China. Tel: 8613637312158 e-mail: yintianm@126.com

O2016 Turkish League Against Rheumatism. All rights reserved. 
contained 230 cells/uL (124 lymphocytes and 106 neutrophils) and $852.8 \mathrm{mg} / \mathrm{L}$ protein. Ink, gram and acid-fast staining of his CSF were negative. Cultures of his CSF yielded no bacterial growth. Cranial magnetic resonance imaging and magnetic resonance angiography and venography showed no abnormalities. $\mathrm{T}_{2}$-weighted spinal magnetic resonance images (Figure 1) showed hyperintensities within the entire spinal cord. $\mathrm{He}$ was diagnosed with longitudinal myelitis associated with NBD.

Intravenous methylprednisolone (120 mg/day) was administered for three days, followed by an oral administration of prednisolone $(45 \mathrm{mg} /$ day). In conjunction with the steroid therapy, intravenous cyclophosphamide $(0.4 \mathrm{~g})$ was administered twice. There was rapid regression in his complaints after half a month of therapy. He was able to control his bladder and had increased muscle strength in his lower limbs (phase 4+ in both). He was

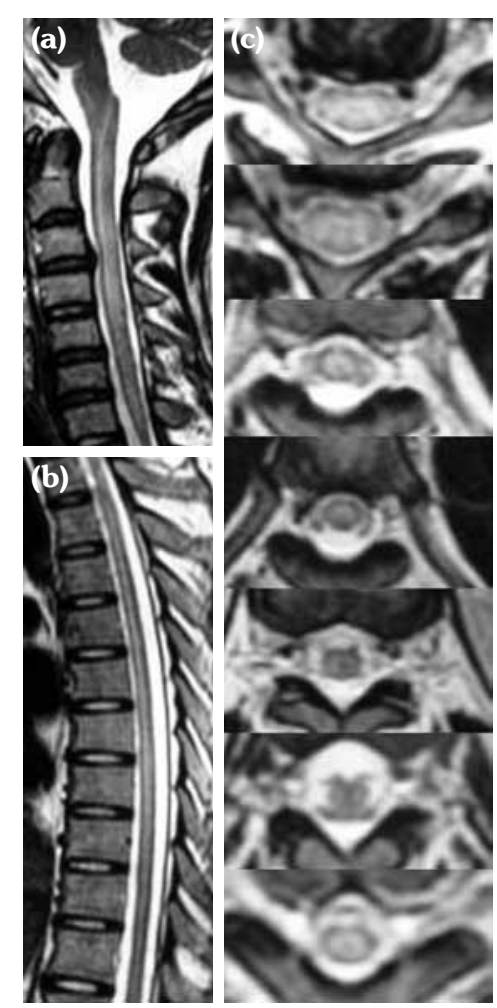

Figure 1. $T_{2}$-weighted spinal cord magnetic resonance image showed hyperintensities within cervical and (a) thoracic and lumbar (b) spinal cord. Axial images revealed hyperintensity in spinal cord at different levels (c). able to walk by himself without any assistance. Routine biochemical tests showed decreased erythrocyte sedimentation rate $(4 \mathrm{~mm} / \mathrm{h})$ and C-reactive protein $(6 \mathrm{mg} / \mathrm{L})$. Cells and protein in CSF decreased to $20 / \mathrm{uL}$ and $311.5 \mathrm{mg} / \mathrm{L}$, respectively. The patient was neurologically stable after three months of follow-up.

\section{DISCUSSION}

This patient fulfills diagnostic criteria for Behçet's disease. $^{3}$ In NBD, the central nervous system might be involved in one or both of two ways: first, through the development of an immune mediated meningoencephalitis, and second, as a consequence of thrombosis within the dural venous sinuses. ${ }^{4}$ The most common manifestations of NBD are fever, headache, motor weakness, and cranial nerve palsy and there is a low prevalence of behavioral changes, seizures, and sphincter incontinence. Prognosis of NBD is far from optimistic. ${ }^{4}$ For patients with poor prognostic factors such as multifocal involvement, spinal presentations, more than two attacks per year, progressive course, and increased CSF cell count and protein content at the time of neurologic manifestations, intravenous cyclophosphamide and corticosteroids are recommended. ${ }^{5}$ If these regimens fail, biologics should be added. ${ }^{6}$ Most nonresponders to cyclophosphamide and corticosteroids are recalcitrant or refractory cases. $^{7,8}$ This high-risk patient responded well to the immunosuppressive therapy approximately in a period of 15 days. To the best of our knowledge, in the literature, there is another case of NBD with longitudinally extensive transverse myelitis who achieved marked clinical improvements after treatment with intravenous and oral steroids followed by six months of pulsed intravenous cyclophosphamide. ${ }^{9}$

Spinal cord involvement in NBD is not frequently encountered, and longitudinal myelitis is rare, which has to be differentiated from other pathologies such as multiple sclerosis, central nervous system lupus, acute disseminated encephalomyelitis, central nervous system lymphoma, and neuromyelitis optica. ${ }^{10,11}$

Neuro-Behçet's disease associated longitudinal myelitis is really rare. Magnetic resonance imaging is useful for its diagnosis. Corticosteroid and 
cyclophosphamide are effective in the treatment of longitudinal myelitis in NBD patients.

\section{Declaration of conflicting interests}

The authors declared no conflicts of interest with respect to the authorship and/or publication of this article.

\section{Funding}

The authors received financial support from the project of Natural Science Foundation of China (No. 81401356) for this article.

\section{REFERENCES}

1. Sakane T, Takeno M, Suzuki N, Inaba G. Behçet's disease. N Engl J Med 1999;341:1284-91.

2. Kidd D, Steuer A, Denman AM, Rudge P. Neurological complications in Behçet's syndrome. Brain 1999;122:2183-94.

3. Criteria for diagnosis of Behçet's disease. International Study Group for Behçet's Disease. Lancet 1990;335:1078-80.

4. Al-Araji A, Kidd DP. Neuro-Behçet's disease: epidemiology, clinical characteristics, and management. Lancet Neurol 2009;8:192-204.

5. Borhani Haghighi A. Treatment of neuro-Behçet's disease: an update. Expert Rev Neurother
2009:9:565-74.

6. Fasano A, D'Agostino M, Caldarola G, Feliciani C, De Simone C. Infliximab monotherapy in neuro-Behçet's disease: four year follow-up in a long-standing case resistant to conventional therapies. J Neuroimmunol 2011;239:105-7.

7. Licata G, Pinto A, Tuttolomondo A, Banco A, Ciccia $\mathrm{F}$, Ferrante $\mathrm{A}$, et al. Anti-tumour necrosis factor alpha monoclonal antibody therapy for recalcitrant cerebral vasculitis in a patient with Behçet's syndrome. Ann Rheum Dis 2003;62:280-1.

8. Fujikawa K, Aratake K, Kawakami A, Aramaki T, Iwanaga $\mathrm{N}$, Izumi $\mathrm{Y}$, et al. Successful treatment of refractory neuro-Behcet's disease with infliximab: a case report to show its efficacy by magnetic resonance imaging, transcranial magnetic stimulation and cytokine profile. Ann Rheum Dis 2007;66:136-7.

9. Graham D, McCarthy A, Kavanagh E, O'Rourke K, Lynch T. Teaching Neurolmages: longitudinally extensive transverse myelitis in neuro-Behcet disease. Neurology 2013;80:189-90.

10. Trebst C, Raab P, Voss EV, Rommer P, Abu-Mugheisib $\mathrm{M}$, Zettl UK, et al. Longitudinal extensive transverse myelitis-it's not all neuromyelitis optica. Nat Rev Neurol 2011;7:688-98.

11. Nardone R, Fitzgerald RT, Bailey A, Zuccoli G. Longitudinally extensive transverse myelitis in systemic lupus erythematosus: case report and review of the literature. Clin Neurol Neurosurg 2015;129:57-61. 